\section{Spontaneous intracranial hypotension: case report}

\author{
P. Merlo (凶) • A.M. Clerici • B. Stival \\ V. Rebecchi • M. Noce $\cdot$ S. Mazza \\ U.O. Neurologia, \\ Istituto Clinico Humanitas, \\ Rozzano, Milan, Italy \\ E-mail: paola.merlo@umanitas.it \\ Tel.: +39-02-82244628 \\ Fax: +39-02-82244693
}

Published online: 15 December 2005

\begin{abstract}
Spontaneous intracranial hypotension is a well defined entity, however it is characterised by a relevant clinical heterogeneity. The main feature is orthostatic headache, often accompanied by other symptoms. The diagnosis is easily made in the presence of orthostatic headache, excluding an obvious aetiology of low cerebrospinal fluid (CSF) pressure, and often there is no evidence of direct or indirect CSF leak.
\end{abstract}

Keywords Headache • Intracranial hypotension

\section{Case report}

A 35-year-old woman complained, for 10 days, of a sudden and severe occipitonucal throbbing pain radiating to both temporal sites, along cervico-dorsal spine. Headache was relieved in the lying-down position and worsened in a sitting or standing position, but was also associated with other symptoms such as diplopia and dizziness. Her anamnesis was negative for any medical illness, previous trauma, lumbar puncture or neurosurgery treatment. Physical examination was normal; neurological examination only showed VI cranial nerve palsy in right eye and neck stiffness. Brain and cervicodorsal magnetic resonance imaging (MRI) showed a diffuse meningeal enhancement extending into the cervical region and a fluid collection in the cervical and dorsal extradural space, while the lumbosacral MRI and angio-MR were normal. Blood chemistry and routine tests were normal, including inflammatory-neoplastic-autoimmune-thyroid assessment. A neuroradiological examination did not show any cerebrospinal fluid (CSF) leakage. Also radioisotope cisternography was performed and no evidence of CSF direct or indirect leak, abnormal CSF flow or ventricular reflux was noted. Starting treatment with strict bed rest, a course of methylprednisolone and forced saline hydration, the headache disappeared after 2 weeks and after one month the patient was totally asymptomatic. Brain and cervical MRI normalised after 6 months. Clinical and neuroradiological observation a year later were negative.

\section{Discussion}

Spontaneous intracranial hypotension $(\mathrm{SIH})$ is a rare but well known entity, described for the first time by Schaltenbrand in 1938 [1] and actually classified in the International Classification of Headache Disorders 2nd Edition (ICHD-II) in 2004. The clinical picture is very heterogeneous, including a not always orthostatic headache that may be associated with one or more several other symptoms, including pain or stiffness of the neck, nausea, horizontal diplopia, dizziness, visual blurring and photophobia [2]. The diagnosis of SIH is generally one of exclusion, so that precipitating events such as recent operative procedures, lumbar puncture or traumatic dural tear and other medical conditions (severe systemic infection, diabetic coma, administration of hypertonic solution, hyperpnoea) must be ruled out [3]. Diagnosis can be made by many examinations, including lumbar puncture, however is substantially based on the clinical data, supported by the role of neuroimaging (MRI with gadolinium, cisternography or CT-myelography). The pathophysiology remains unclear and treatment may prove difficult in some cases [4]. Treatment is initially conservative, but there is no standard approach, above all based on the prior experiences with post-puncture headache. Generally, good results derives from bed rest, hyperhydration and corticosteroids [5].

\section{References}

1. Schaltenbrand G (1938) Neure anschauungen zur pathophyiologie der liquorzirkulation. Zentralbl Neurochir 3:290-300

2. Silberstein SD, Lipton RB, Goadsby PJ (2002) Headache in clinical practice. Isis Medical Media, Oxford

3. Marcelis J, Silberstein SD (1990) Spontaneous low cerebrospinal fluid pressure headache. Headache 30:192-196

4. Fernandez E (1990) Headache associated with low spinal fluid pressure. Headache 30:122-128

5. Mokri B (2005) Spontaneous intracranial hypotension spontaneous CSF leaks. Headache Currents 2:11-22 